Chapter 9

\title{
Promotion of Blood Fluidity Using Electroacupuncture Stimulation
}

\author{
Shintaro Ishikawa, Kazuhito Asano and \\ Tadashi Hisamitsu \\ Additional information is available at the end of the chapter \\ http://dx.doi.org/10.5772/53036
}

\section{Introduction}

Acupuncture is an alternative medicine originating in ancient China that treats patients by manipulating thin, solid needles that have been inserted into acupuncture points in the skin. Current several scientific research supports acupuncture's efficacy in the relief of certain types of pain and post-operative nausea [1,2].

Acupuncture's greatest effectiveness appears to be in symptomatic control of pain and nausea. The World Health Organization and the United States' National Institutes of Health (NIH) have stated that acupuncture can be effective in the treatment of neurological conditions and pain [3]. Moreover, it is thought that acupuncture regulates various biological functions. It was presented that acupuncture stimulus influences the cytokine level, hormone level [4] and leukocyte number [5] as effects for blood.

Blood roles in organisms include waste product removal, body temperature adjustment, as well as oxygen and nutritive supply. Physiologically the first priority driving force of blood circulation is cardiac pressure.

Blood flow is determined by co-action of the cardiovascular system and blood fluidity. However, blood flow is also controlled by a blood hydrodynamic characteristic. It is established that changes in the cardiovascular system will cause changes in blood properties as well [6-9]. Changes of blood cell composition and plasma components may influence blood fluidity in the long term [7], and blood cell activity, such as red blood cell agglutination, leukocyte adherence, and platelet aggregation, in the short term $[8,9]$.

It is believed that variations in blood fluidity result in disorders of the circulatory system such as arterial sclerosis or embolism, damage to vascular endothelium cells by hyperten- 
sion, glucose tolerance degradation and chronic inflammation, degradation of blood vessel flexibility by hyperlipemia and aging, weakness of blood cells, and degradation of plasma plasticity $[10,11]$.

Recently it is assumed that cerebral infarction, myocardial infarction, and pulmonary infarction are caused by an increase of thrombus generation. Common treatments and recurrence prevention for these illnesses include administration of antithrombotic agent such as warfarin and aspirin [12,13].

In the world today Western medicine is sometimes complemented with alternative methods of treatment although this trend is somewhat stalled due to the lack of the scientific evidence for the efficacy of alternative medicine. Western medicine has shown to have a direct effect on cell and metabolic function.

In oriental medicine, the state in which blood is stagnant is called "oketsu (Yu xie)," meaning preceding state or symptomatic of sickness. Acupuncture stimulation is often clinically used for treatment of "oketsu." The degree of "oketsu" is indicated by tongue color and form, swelling, paroxysmal blushing, and dark circles under the eyes [14]. However, these indexes do not reflect the real hemogram.

"oketsu" is now regarded as physiological blood flow and is studied from the point of view of blood fluidity and vascular resistance. We previously reported $[15,16]$ that (1) various types of stress applied to rats cause blood fluidity to decline drastically, as the platelet adhesion increases, (2) acupuncture stimulation increases blood fluidity by the depression of platelet adhesion, and (3) the effects of acupuncture stimulation vary according to the stimulus location (acupoint). Thus, acupuncture stimulus were much effective to improve the decrease of blood fluidity such as "oketsu." The mechanisms that interact between acupuncture stimulus and blood fluidity, however, have not yet been identified.

In this chapter, we first describe our original finding on these topics, and then propose the possible action mechanisms of the improvement of blood fluidity by electroacupuncture.

\section{Materials \& methods}

\subsection{Experimental animals}

Specific pathogen-free 7-8 weeks old male Wister rats were purchased from Japan Bio-Supply Center (Tokyo, Japan). The animals were maintained at $25 \pm 2{ }^{\circ} \mathrm{C}$, humidity $55 \pm 5 \%$, and a light and dark cycle of 12 hour in our animal facilities. The rats were randomly divided into control group or each experiment group, fed a regular show diet and water during experiments. Each group has 7 animals..

\subsection{Acupuncture stimulus}

The modality of acupuncture needle used was $0.20 \times 40 \mathrm{~mm}$ (SEIRIN Co. Japan). Punctures were pricked at acupoints to apply the needle equivalency locus of humans (Figure 1): Zu- 
sanli (ST-36) on the outside crus superior, Sanyinjiao (SP-6) on the inside crus lower part, Hegu (LI-4) between the thumb and the forefinger of the arm, Neiguan (P-6) on the distal inside arm, and Shenshu (BL-23) on the outside dorsum spine. Acupuncture was $5 \mathrm{~mm}$ deep and stimulated electrically $(3-5 \mathrm{~V}, 30-200 \mu \mathrm{A}$, rectangular and bi-phasic) at a stimulation frequency of 1 or $100 \mathrm{~Hz}$ to permit the muscle to shrink slightly. An Ohm Palser LFP-4000A (Zen Iryoki Co. Japan) was used as the device of acupuncture stimulus.

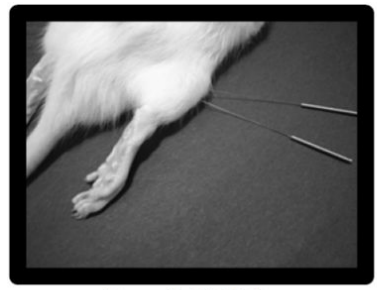

Zusanli (ST-36)

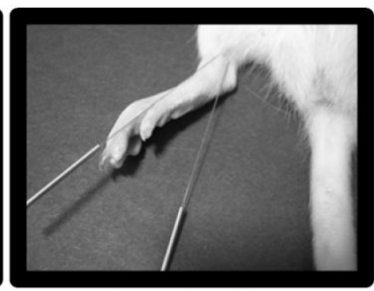

Sanyinjao (SP-6)

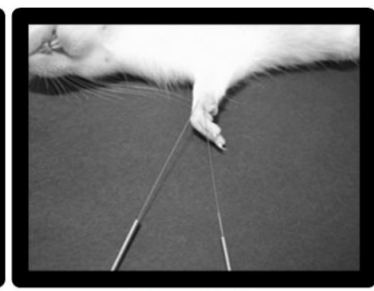

Hegu (LI-4)

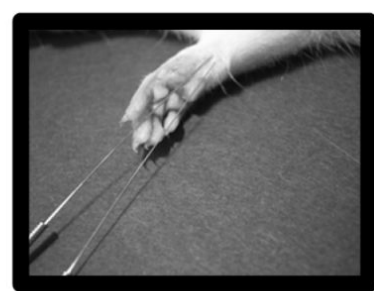

Neiguan (P-6)

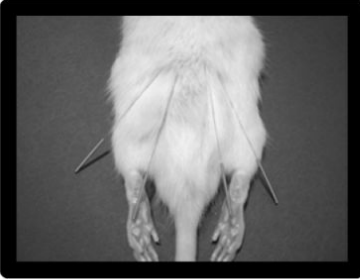

Shenshu (BL-23)

Figure 1. Locations of the acupoints. Cited from [15], with permission

\subsection{Measurement of blood fluidity}

We determined the blood fluidity using a Micro Channel array Flow Analyzer KH-6 (MCFAN; MC Laboratory Inc. Japan). Coagulation of blood was blocked by Heparin sodium. We used $100 \mu \mathrm{l}$ of the blood to measure the flow time to the silicon tip of the analyzer (Figure 2)

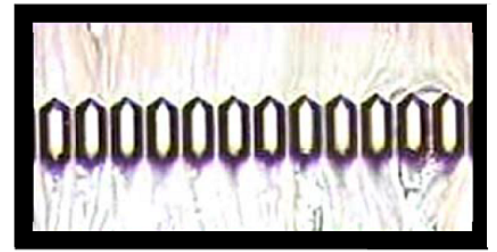

A. Blood with high fluidity

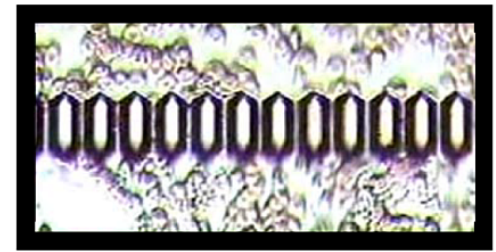

B. Blood with low fluidity

Figure 2. Images of blood flow by MC-FAN MC-FAN measures passing time of blood (100 $\mu \mathrm{L})$.A. Blood flow velocity is rapid. Blood cells are not observed. B. Blood flow velocity is slow. Platelet agglutination is observed. 
We assumed that the flow time in the analyzer imitated the capillary blood fluidity index [17]. In an MC-FAN assay, the prolongation of flow time indicates a decrease of blood fluidity and a short flow time, an increase of blood fluidity [18].

\subsection{Measurement of platelet aggregation ability}

The ability of platelet aggregation was measured with a platelet coagulation measuring system, a platelet aggregometer (PA-20; Kowa Company. Ltd., Tokto, Japan). Blood samples were collected from the inferior vena cava under pentobarbital anesthesia. The blood sample treated with $3.2 \%$ of sodium citrate $(2.0 \mathrm{~mL})$ was centrifuged to obtain platelet-rich plasma (PRP) and platelet-poor plasma (PPP).

PRP in a cuvette was pre-warmed to $37^{\circ} \mathrm{C}$; adenosine $5^{\prime}$-diphosphate (ADP) was added as an agonist and the aggregation level was measured. This PA-20 device can measure platelet aggregation by the light scattering method. This light scattering method can measure the platelet aggregate size by determining the intensity of scattered light emitted from a particle, the light intensity directly corresponding to the particle size. The platelet aggregation curve was separately recorded for each size range as the voltage of light scattering intensity. The aggregates measured were divided into three categories according to size: small (diameter 9-25 $\mu \mathrm{m}$ ), medium (diameter 26-50 $\mathrm{m}$ ), and large-sized aggregates (diameter 50-70 $\mu \mathrm{m}$ ) $[19,20]$.

\subsection{Affirmation of blood cell count}

In blood fluidity experiments, it is important to consider factors which influence blood properties: the number of erythrocytes, leukocytes, platelets and hematocrit.

Therefore, we confirmed that the above-mentioned blood properties showed no significant differences in both the experiment and control groups.

\subsection{The experimental profile}

This experiment is divided into three parts. The first is the examination of modality of acupuncture stimulus. The second is the examination of action mechanisms. The third examination focuses on the decreasing state of blood fluidity by the restraint stress method. And finally we reviewed whether acupuncture improves blood fluidity.

\section{Results \& discussion}

\subsection{Effects of stimulus approach}

At first, blood fluidity was reviewed in several acupuncture stimulus approaches (Figure 3). 


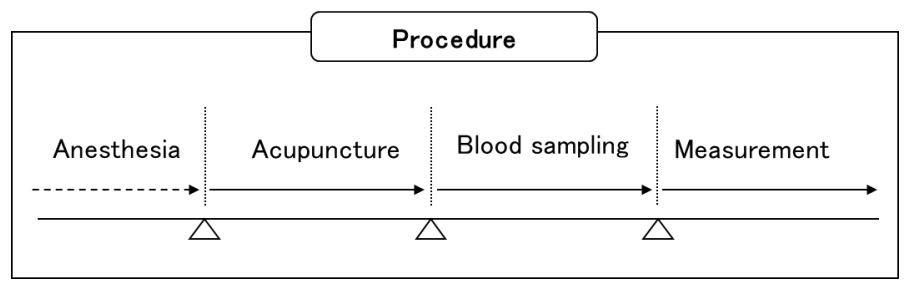

Figure 3. Procedure of study 1-7

\subsubsection{Study 1. The influence of prickle acupuncture locus}

We applied acupuncture stimulation to determine the influence of acupuncture stimulus on blood fluidity for 60 minutes for only 1 day at $1 \mathrm{~Hz}, 3-5 \mathrm{~V}$ [21]. Acupuncture was performed under anesthesia. Blood samples were collected from the abdominal vein after acupuncture stimulus. The blood samples were pretreated with anticoagulant (heparin sodium). The control group was anesthetized the same as the experimental groups but did not receive stimulation. Because the effects of acupuncture vary according to stimulus locus (acupoint), acupuncture was applied to various loci of the trunk, arm and lower extremities. Results showed that blood flow time shortened significantly in the group with stimulation to the Zusanli, Hegu and Sanyingao; however, there were no significant changes in the group with stimulation to Neiguan and Shenshu acupoints.

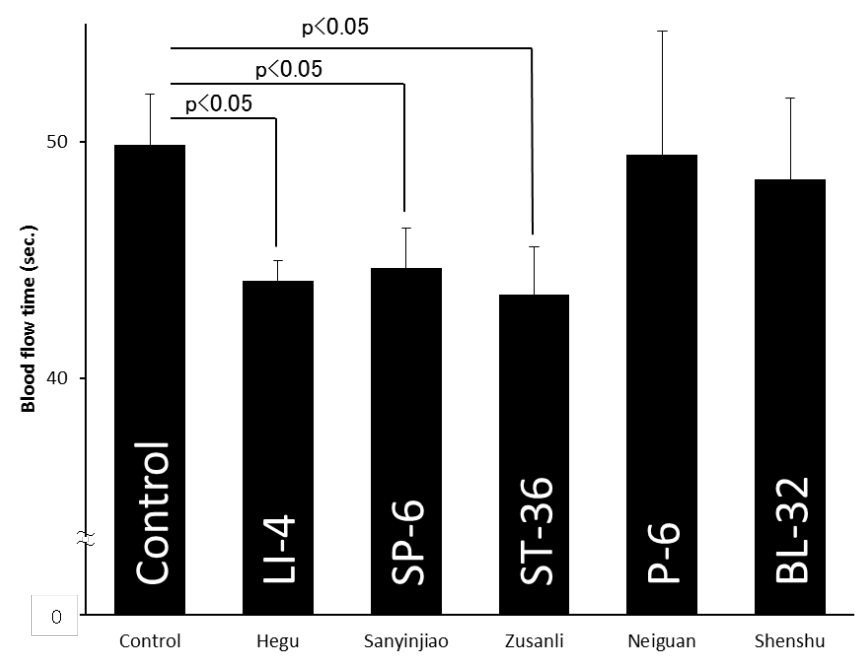

Figure 4. The influence of prickle acupuncture locus 
Figure 4 shows that when acupuncture stimulation was applied to the Hegu, Sanyinjao and Zusanli acupoints, blood fluidity was enhanced in comparison with the control. In addition, the results reveal that blood fluidity is not altered by stimulation of any of the acupoints in this study suggesting specificity of acupoints contributing to blood fluidity. Heparin sodium is combined with antithrombin III and inhibits thrombin activity, coagulation factor Xa and XIIa. In other words heparin sodium does not inhibit agglomeration of platelets directly. When heparin was used as an anticoagulant, MC-FAN blood fluidity observation showed the influence of platelet aggregation ability and erythrocyte deformability.

\subsubsection{Study 2. The influence of stimulus time}

This shows the effect of differences of stimulation period (Figure 5). Acupuncture was stimulated for 15 or 60 minutes at $1 \mathrm{~Hz}, 3-5 \mathrm{~V}$ to the Zusanli acupoint under anesthesia. Acupuncture stimulation by Zusanli enhanced the blood fluidity (reduced the blood flow time (Figure 4). Blood samples were collected from the abdominal vein after acupuncture stimulus. The blood samples were preprocessed with an anticoagulant (heparin sodium).

The control group was anesthetized in the same manner as the experimental groups but did not receive stimulation.

As shown in this result, both stimulation time of 15 minutes and 60 minutes represented similar effect. Even with a short stimulation time, blood fluidity was enhanced suggesting the immediate effect of acupuncture stimulation contributing to blood fluidity.

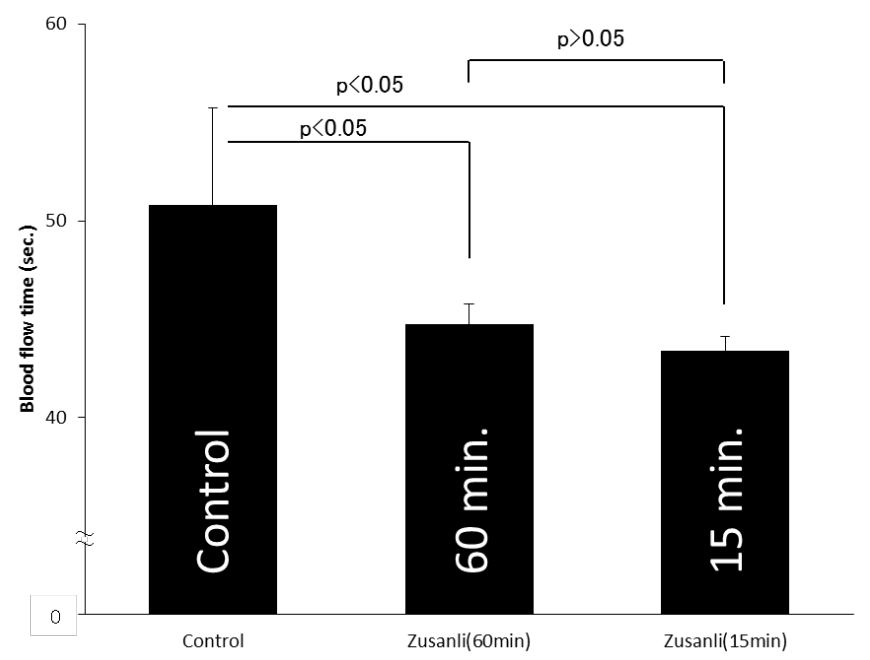

Figure 5. The influence of stimulus tome on blood fluidity. Cited from [16], with permission. 


\subsubsection{Study 3. The influence of stimulus frequency}

The influence of stimulation frequency was investigated (Figure 6). Acupuncture was stimulated for for 60 minutes at $1 \mathrm{~Hz}$ or $100 \mathrm{~Hz}, 3-5 \mathrm{~V}$ to Zusanli acupoint under anesthesia. When acupuncture stimulus frequency changed, the blood fluidity was enhanced at both $1 \mathrm{~Hz}$ and 100 $\mathrm{Hz}$. This result indicates that blood fluidity is not affected by change of stimulus frequency.

It is known that a low-frequency $(1-2 \mathrm{~Hz}$ ) or a high-frequency (more than $100 \mathrm{~Hz}$ ) stimulus influences mechanisms other than those of acupuncture analgesia [22]. When these two acupuncture analgesic system influences, even an operation is enabled only by acupuncture anesthesia. We think that a decrease of nociception affects blood fluidity. Electric acupuncture of low frequency stimulus (1-5 Hz) secretes arterenol, serotonin and $\beta$-endorphin in the central nervous system. In other words, it is thought that the secretion of these transmitters has analgesic and sedative effects on the descending pain modulatory system or the endogenic opioid system [22-24]. In addition, as mechanism through the endogenous opioid system, precedence study shows acupuncture analgesic system of diffuse noxious inhibitory controls (DNIC) participated in acupuncture and moxibustion induced-analgesia [25]. In addition, spinal segment-related analgesia occurs at high-frequency (more than $100 \mathrm{~Hz}$ ) electric acupuncture. This analgesic system produces an analgesic effect in concurrence with the start of the stimulus. It is thought that the gate control theory applies because this system does not compete with naloxone administration [23,24,26,27].

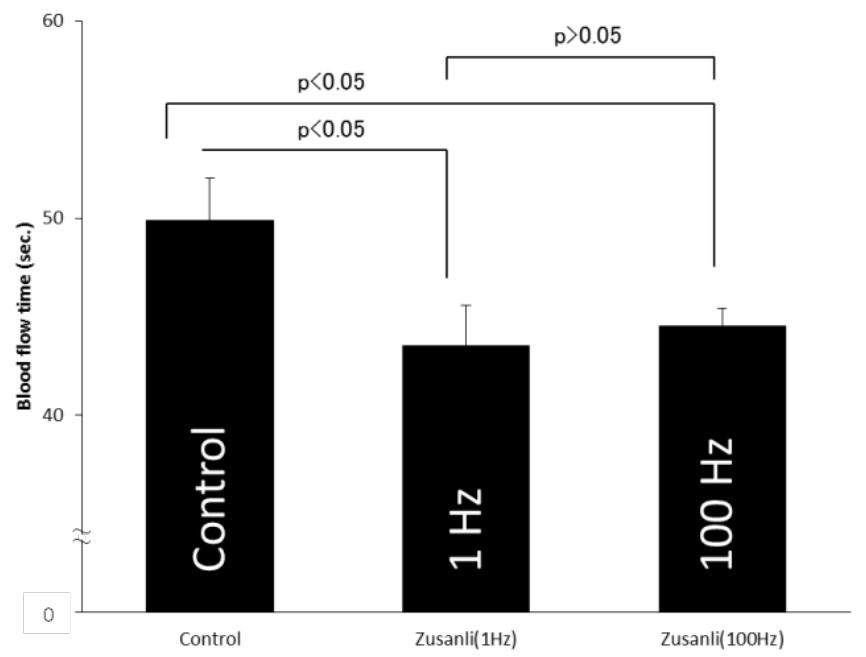

Figure 6. The influence of stimulus frequency on blood fluidity. Cited from [16], with permission. 


\subsection{Effects of action mechanism}

In the second set of experiments, we reviewed the mechanism of acupuncture with medicine.

\subsubsection{Study 4. Influence of naloxone}

The influence of Naloxone, an antagonist of endogenous opioid was investigated (Figure 7). Acupuncture on the Zusanli was stimulated 60 minutes while injecting naloxone $(5 \mathrm{mg} / \mathrm{kg}$, i.p.) into the abdominal cavity every 10 minutes.

Neither acupuncture stimulus nor naloxone was given to the control groups. Physiological saline was injected into the abdominal cavity every 10 minutes of the only acupuncture and the control groups. Results showed that blood flow time decreased significantly in the $\mathrm{Zu}$ sanli-stimulated and the Zusanli-stimulated plus naloxone groups, although there was no significant difference between these two groups.

The results of figure 5 show that blood fluidity changes with short time electro acupuncture suggesting the intervention of the nervous system. However, when we consider the fact that blood fluidity was not affected by a difference of stimulus frequency nor naloxone administration, it can be surmised that the endogenic opioid system and the spinal segment system do not contribute to blood fluidity. We speculate that acupuncture stimulus changes blood fluidity by the automatic nervous system and axon reflex, and does not influence the opioid system and the spinal segment analgesia system.

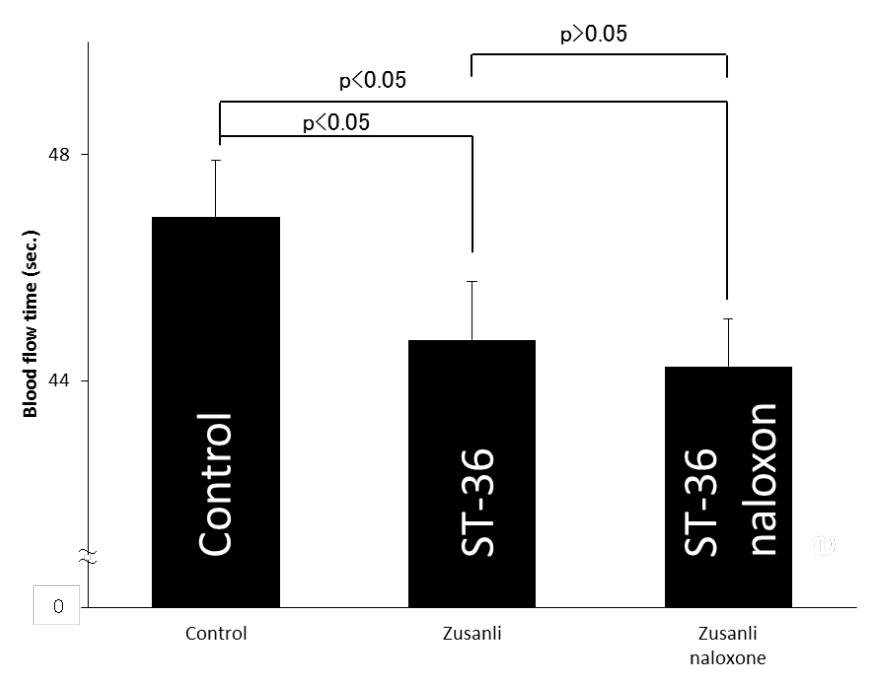

Figure 7. Influence of naloxone on blood fluidity. Cited from [16], with permission. 


\subsubsection{Study 5. Influence of $\beta$ antagonist}

A. The effect of intraperitoneal administered adrenergic drugs on blood fluidity

The influence of sympathetic agonists and antagonists on the blood flow was investigated (Figure 8). We used phenylephrine at $200 \mu \mathrm{g} / \mathrm{kg}$ as an $\alpha$-agonist, phentolamine at $100 \mu \mathrm{g} / \mathrm{kg}$ as an $\alpha$-antagonist, isoproterenol at $4 \mu \mathrm{g} / \mathrm{kg}$ as a $\beta$-agonist, and propranolol at $40 \mu \mathrm{g} / \mathrm{kg}$ as a $\beta$-antagonist. In these experiments, the drugs were dissolved in $1 \mathrm{~mL}$ of physiological saline and were administered by intraperitoneal (i.p.) into the rats. Physiological saline (1 mL) was administered by i.p. to the control animals.

The blood flow time was increased with $\alpha$-agonist and $\beta$-antagonist, and the blood flow time was decreased with $\beta$-agonist and $\alpha$-antagonist.

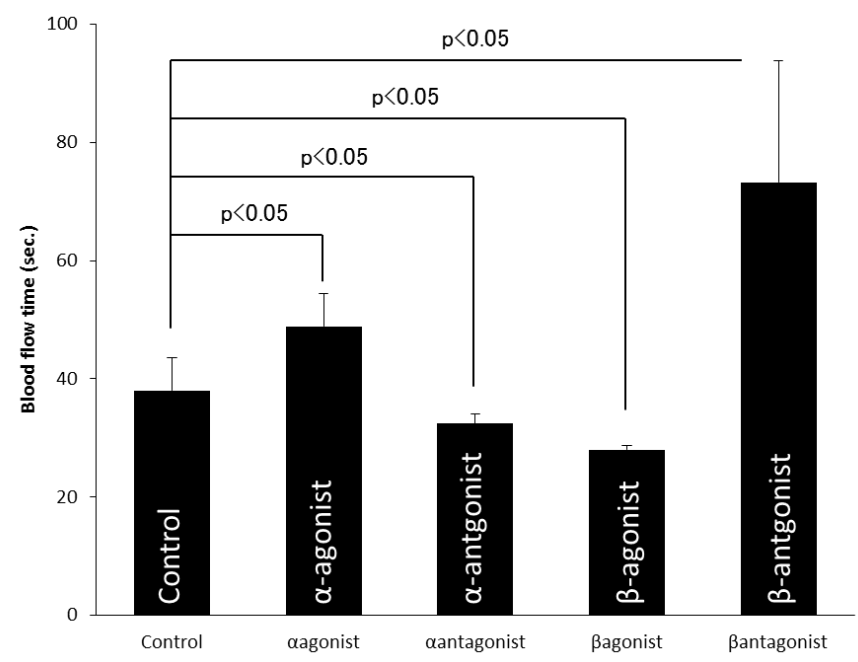

Figure 8. Influence of sympathetic agonists and antagonists on blood fluidity. Cited from [16], with permission.

B. Effect of $\beta$-antagonist on the blood fluidity enhanced by acupuncture

Effect of acupuncture together with an adrenergic drug on the blood fluidity was investigated to get the insight into the reaction mechanism (Figure 9). $\beta$-antagonist, propranolol at 40 $\mu \mathrm{g} / \mathrm{kg}$, was dissolved in $1 \mathrm{~mL}$ of physiological saline and administered before the acupuncture stimulation by i.p into the rats. Acupuncture was stimulated at $1 \mathrm{~Hz}, 3-5 \mathrm{~V}$ for $60 \mathrm{mi}$ nutes to Zusanli acupoint under anesthesia. 


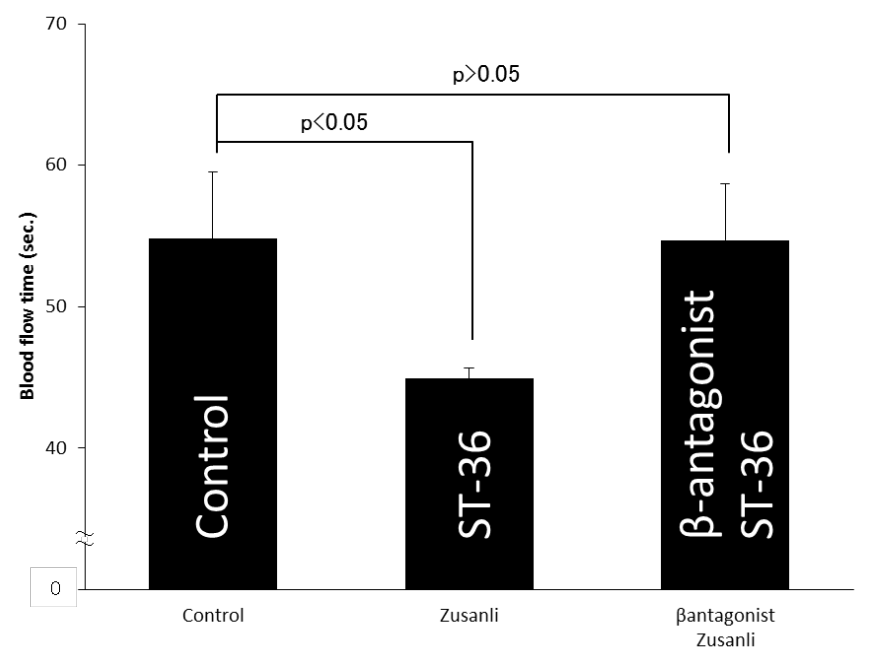

Figure 9. The relevance with ßantagonist and blood fluidity. Cited from [16], with permission.

As a result, the $\beta$-antagonist canceled the enhancement effect of blood fluidity with acupuncture stimulus. There was no significant difference between the $\beta$-antagonist plus acupuncture and the control groups. This result indicates that the sympathetic nervous system influences blood fluidity with an acupuncture stimulus.

\subsubsection{Study 6. Inhibition of platelet aggregation}

Effect of platelet aggregation was investigated (Figure 10). An ADP an agonist was added in the PRP and the aggregation level was measured by the light scattering method. Acupuncture decreased the formation of large aggregates. In addition, medium and small-sized aggregates in the stimulated experimental group increased significantly. However, in animals previously administered with $\beta$-antagonist, the decrease of platelet aggregation by acupuncture was significantly reversed. This result indicates that platelet aggregation may contribute to the blood fluidity enhanced by acupuncture.

\subsubsection{Study 7. Influence of acupuncture and $\beta$-antagonist on the platelet aggregation}

Erythroid deformability was measured by blood fluidity using erythrocyte suspension (Figure 11). Physiological saline $(2.0 \mathrm{ml})$ was added to the blood sample treated with heparin sodium $(2.0 \mathrm{ml})$ and centrifuged $(400 \mathrm{~g} \times 5$ minutes $)$, resulting in a deposition layer of erythrocyte suspension and removal of the supernatant. Phosphate-buffered saline (PBS) was added to this erythrocyte rich liquid and re-centrifuged twice in the same manner [28]. The layer of erythrocyte was adjusted to $30 \%$ with hematocrit, and this erythrocyte suspension was used for this experiment. The erythrocyte suspension was examined with the MC-FAN. 
The fluidity of erythrocyte suspension was not altered by acupuncture. (Figure 11), indicating that erythrocyte deformability does not contribute to blood fluidity strengthened by acupuncture.

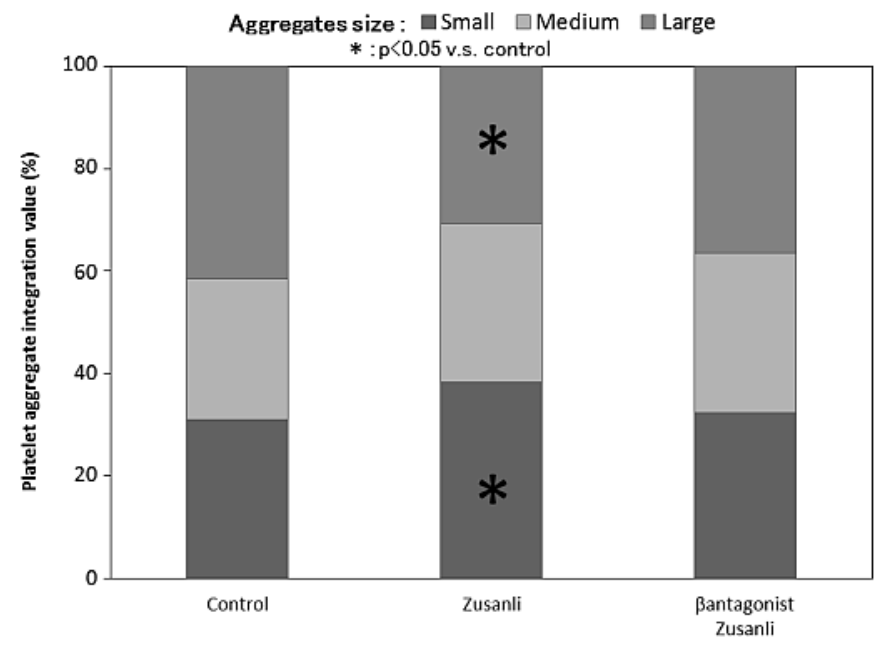

Figure 10. Observation of platelet aggregation Cited from [16], with permission

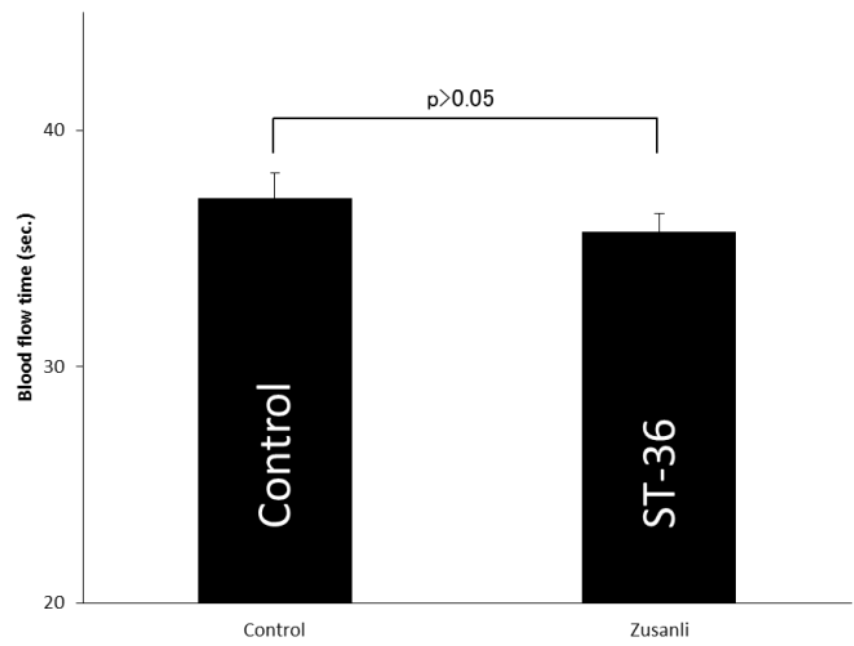

Figure 11. Examination of erythrocyte suspension fluidity. Cited from [15], with permission. 


\subsection{Influence of restraint stress}

Finally, we also focused on the acupuncture stimulus mechanism with the restraint stress method. As preliminary research, we hypothesized that various stressors decrease blood fluidity. Restraint in a rectangular acrylic box for six hours was used as the stressor. Acupuncture stimulation was given for 1 hour (after 5 hours of restriction) at $1 \mathrm{~Hz}, 3-5 \mathrm{~V}$ to the Zusanli acupoint with arousal (Figure 12).

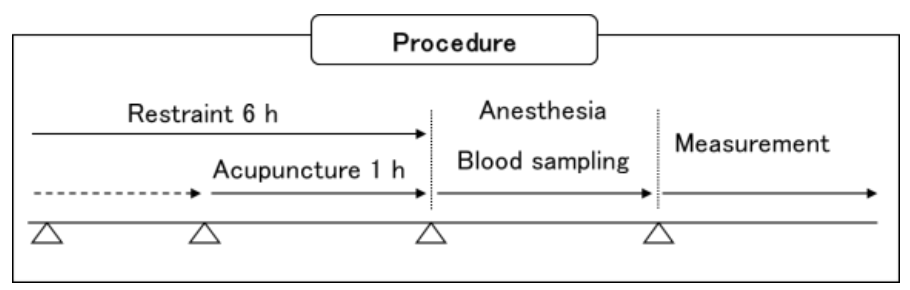

Figure 12. Experimental procedures for the study 8 and 9

\subsubsection{Study 8. Observation of blood fluidity and platelet aggregation}

Effect on the blood fluidity and platelet aggregation was investigated (Figure 13). In the restraint group, the blood fluidity decreased and the platelet aggregation increased. However, the acupuncture stimulus reversed these changes.
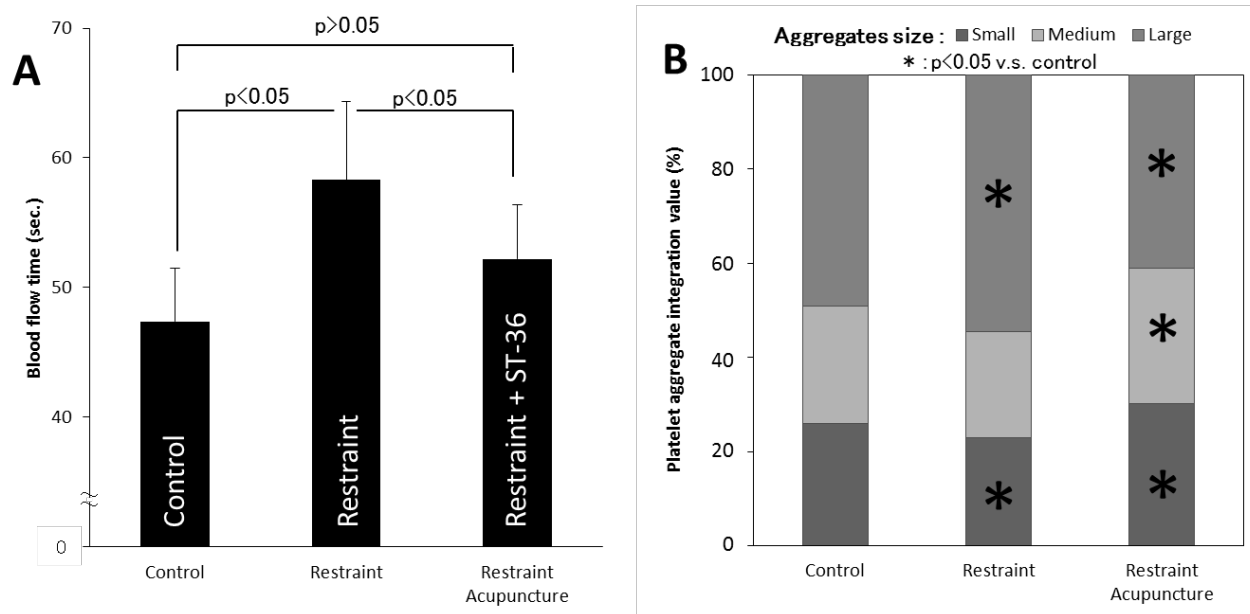

Figure 13. Influence of restraint stress on the bloody fluidity (A) and platelet aggregation (B) 


\subsubsection{Study 9. Affecters that are involved in platelet aggregation}

(a) Blood noradrenalin level

Changes in the blood noradrenaline level after restraing stress and acupuncture was monitored by 2-CAT (A-N) Research ELISA (Labor Diagnostika Nord GmbH \& Co. KG) (Figure 14).

In the restraint group, the blood noradrenalin level increased. In the restraint and acupuncture group, the adrenalin level decreased. These results suggest that acupuncture stimulus inhibits noradrenalin release.

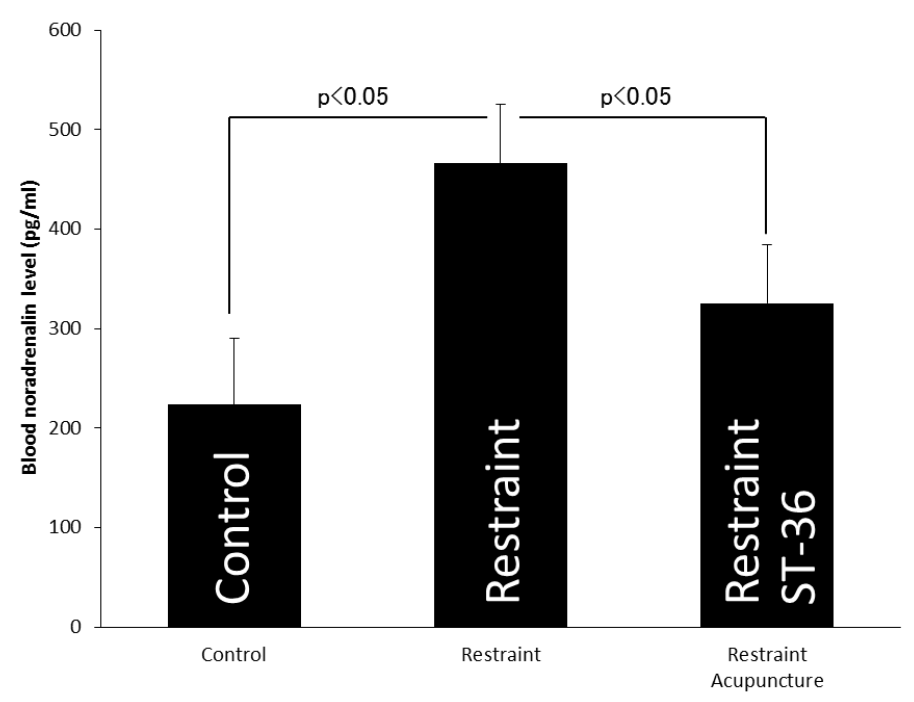

Figure 14. Blood noradrenalin level

(b) Blood ATP level

Changes in the blood ATP level after restraint and acupuncture was monitored by AMERIC ATP kit (Applied Medical Enzyme Research Ins. Co.) (Figure 15).

In the restraint group, the blood ATP level increased. In the restraint and acupuncture group, the ATP level decreased, indicating that acupuncture stimulus most likely inhibits ATP release. 


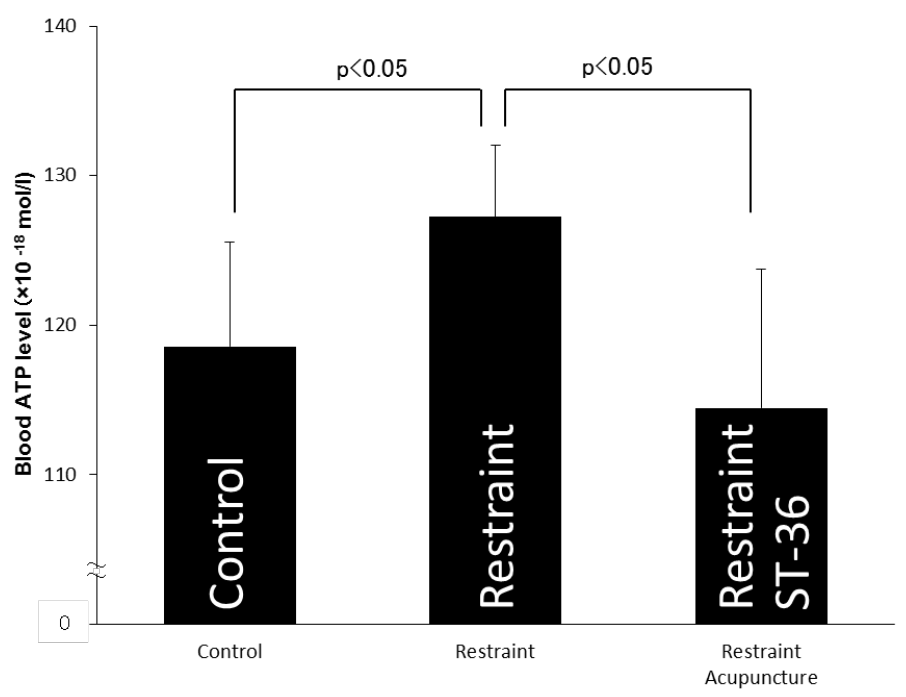

Figure 15. Blood ATP level

(c) Blood NO level

Changes in blood nitric oxide (NO) level after restraint and acupuncture was monitored by Greies test (NO2/NO3 assay kit, Dojindo Molecular Technologies, Inc) (Figure 16). NO is produced by a vascular endothelial cells, and regulates a vascular endothelium function. $\mathrm{NO}$ is a molecular mediator of many physiological processes, including vasodilation, inflammation, thrombosis, immunity and neurotransmission [29,30]. Half-life of NO is very short (for 3-6 seconds). It is thought that $\mathrm{NO}_{2}^{-}$reflects production of NO. Therefore a level of $\mathrm{NO}_{2}{ }^{-}$, which occurred by the oxidation of $\mathrm{NO}$, was examined by the Griess test $\left(\mathrm{NO}_{2} / \mathrm{NO}_{3}\right.$ Assay Kit, Dojindo Molecular Technologies, Inc.).

In the restraint group, the blood $\mathrm{NO}_{2}^{-}$level decreased. In the restraint and acupuncture group, the $\mathrm{NO}_{2}^{-}$level increased. indicating that acupuncture stimulus most likely improves $\mathrm{NO}_{2}^{-}$release.

Stimulation of $\alpha 1$-adrenoceptor with noradrenaline elicits increases in intracellular $\mathrm{Ca}^{2+}$ concentration and induces platelet aggregation and vasoconstriction. Furthermore, high-shearrate conditions activates von Willebrand factor (vWF) in plasma and vascular sub endothelium and produces platelet aggregation [31]. Vascular smooth muscle is controlled between contraction (induced via $\alpha_{1}$-adrenoceptor) and relaxation (induced via $\beta_{2}$-adernoceptor). It seems that platelet aggregation is enhanced as $\alpha_{1}$-adrenoceptor function is more remarkable than $\beta_{2}$-adrenoceptor function. Therefore acupuncture stimulus down-regulates catecholamine secretion from adrenal medulla and may relax with blood vessels. 


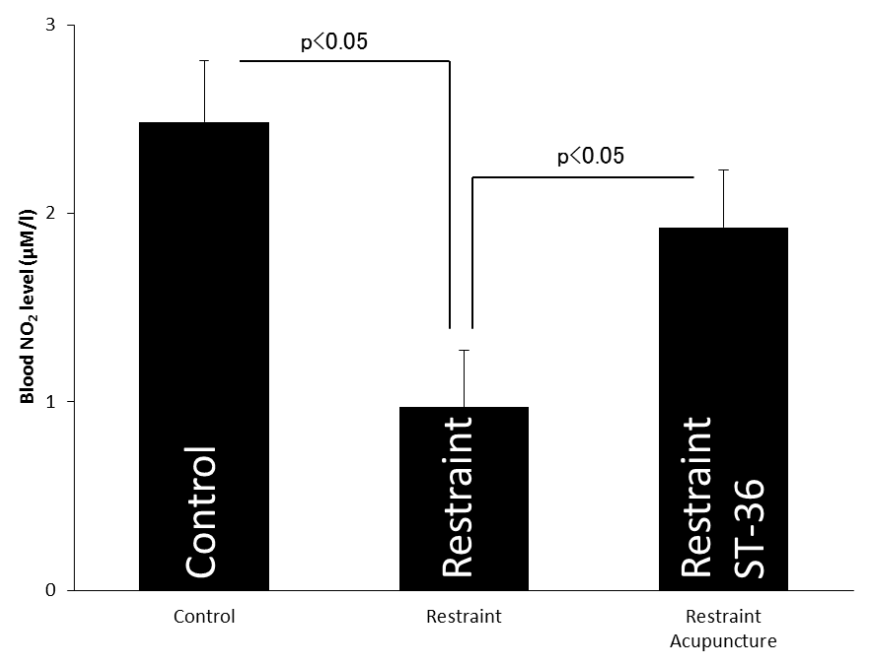

Figure 16. Blood $\mathrm{NO}_{2}$ level

There is widespread appreciation that ATP also acts as an extracellular mediator. The extracellular ATP is released from nerve endings and various secretory cells in an exocytotic manner, from non-excitable cells by mechanical shear stress and from the cytosol of damaged cells. Extracellular ATP activates multiple cell surface P2 receptors and increases intracellular $\mathrm{Ca}^{2+}$, thereby inducing diverse physiological effects, such as regulation of neurotransmitter release, smooth muscle contraction, prostaglandin formation and platelet aggregation [32].

Nitric oxide (NO) which decreases intracellular $\mathrm{Ca}^{2+}$ plays an important role in the regulation of vasoconstriction, inhibition of platelet aggregation, and suppression of smooth muscle cell proliferation. Noradrenalin and ATP increase intracellular $\mathrm{Ca}^{2+}$ in vasucular smooth muscle and platelets [33]. However NO inhibits these functions by depression of intracellu$\operatorname{lar} \mathrm{Ca}^{2+}$.

We think that acupuncture stimulus may control intracellular $\mathrm{Ca}^{2+}$ level of a blood platelet and vasucular smooth muscle generally.

The cardiovascular system are controlled at least in part by blood fluidity. In addition, it is important that blood circulates smoothly for the removal of reactive oxygen and waste products. When pain results from a bruise, distortion and muscle ache, it is known that acupuncture desensitizes the pain. Many preliminary researches have shown that acupuncture inhibits the nerve action of pain.

The present study suggests that acupuncture stimulus changes blood fluidity separately by the cardiovascular system and a new blood flow improvement system that removes pain in response to a lesion, although the precise mechanisms are still a question for future investigation. 


\section{Conclusion}

We conclude that acupuncture stimulation increases blood fluidity. And several points as follows seem to be important to understand the mechanism.

1. There is specificity of stimulus locus.

2. The acupuncture analgesic system seems to be independent from this mechanism that acupuncture increase blood fluidity.

3. Inhibition of the sympathetic nervous activity by the acupuncture stimulation is suggested to have important role in the mechanism.

4. It seems that acupuncture stimulus has a stronger influence on platelets than erythrocytes, and changes blood fluidity.

It is thought that the acupuncture stimulation changes platelet aggregation by various factors, including the presence of catecholamine, ATP and NO.

\section{Author details}

Shintaro Ishikawa ${ }^{1}$, Kazuhito Asano ${ }^{1,2}$ and Tadashi Hisamitsu ${ }^{1}$

1 Department of Physiology, School of Medicine, Showa University, Tokyo, Japan

2 Division of Physiology, School of Nursing and Rehabilitation Sciences, Showa University, Kanagawa, Japan

\section{References}

[1] Lee A, Fan, LTY. Lee, Anna. Stimulation of the wrist acupuncture point P6 for preventing postoperative nausea and vomiting. Cochrane Database of Systematic Reviews (Online) (2): CD003281. doi:10.1002/14651858.CD003281.pub3. 2009

[2] Ernst E, Lee MS, Choi TY. Acupuncture: does it alleviate pain and are there serious risks? A review of reviews. Pain. 152(4):755-64. 2011

[3] McCarthy M. Critics slam draft WHO report on homoeopathy. Lancet, 366: 705-6. 2005.

[4] Sakuma M, Arai M, Matsuba S, Yamaguchi N. Effects of Acupuncture on Intracellular Cytokine and Hormone Levels in Human Peripheral Blood. Cytometry research, 20(1): 41-8, 2010 (in Japanese) 
[5] Yamaguchi N, Hashimoto H, Arai M, Takeda S, Kawada N, Taru A, Li AL, Izumi H, Sugiyama K. Effect of Acupuncture on Leukocyte and Lymphocyte Subpopulation in Human Peripheral Blood: Quantitative discussion. BCPM, 65(4): 199-206, 2002

[6] Hirata C, Kobayashi H, Mizuno N, Kutsuna H, Ishina K, Ishii M. Effect of Normal Human Erythrocytes on Blood Rheology in Microcirculation. Osaka City Medical Journal, 53(2): 73-85, 2007

[7] George JN. Platelets. Lancet, 355: 1531-9, 2000

[8] Fusegawa Y, Hashizume H, Okumura T, Deguchi Y, Shina Y, Ikari Y, Tanabe T. Hypertensive patients with carotid artery plaque exhibit increased platelet aggregability. Thrombosis Research, 117: 615-22, 2006

[9] Lee CY, Kim KC, Park HW, Song JH, Lee CH. Rheological properties of erythrocytes from male hypercholesterolemia. Microvascular Research, 67: 133-8, 2004

[10] Ohno H, Kimu CK, Kim JH, Jung YS, Bae SY, Toshinai K, Miyazaki H, Esaki K, Takemasa T, Kinugasa T, Kizaki T, Hitomi Y, Ookawara T, Oh-Ishi S, Haga S. Hematological Response in Juveniles after Training at Moderate Altitude. Advances in Exercise and Sports Physiology, 10(2): 31-5, 2004

[11] Kikuchi Y. Effect of leukocytes and platelets on blood flow through a parallel array of microchannels: micro- and macroflow relation and rheological measures of leukocyte and platelet activities. Microvasc Res., 50(2): 288-300, 1995

[12] Horn NA, Anastase DM, Hecker KE, Baumert JH, Robitzsch T, Rossaint R. Epinephrine enhances platelet-neutrophil adhesion in whole blood in vitro. Anesth Analg., 100(2): 520-6, 2005

[13] Ochi N, Tabara Y, Igase M, Nagai T, Kido T, Miki T, Kohara K. Silent cerebral microbleeds associated with arterial stiffness in an apparently healthy subject. Hypertension Research, 32(4): 255-60, 2009

[14] Horibe Y, Ishino S, Hisamitsu N, Thein Lain, Ishikawa S, Sato T, Hisamitsu T. "Oketsu" and Hemorheological Changes : Examination by Micro Channel Array Flow Analyzer. Japanese Journal of Oriental Medicine, 55(5): 645-8, 2004 (in Japanese)

[15] Ishikawa S, Murai M, Sato T, Sunagawa M, Tokita E, Aung SK, Asano K, Hisamitsu T. Promotion of blood fluidity by inhibition of platelet adhesion using electroacupuncture stimulation., J Acupunct Meridian Stud., 4(1): 44-53, 2011

[16] Ishikawa S, Suga H, Fukushima M, Yoshida A, Yoshida Y, Sunagawa M, Hisamitsu T. Blood fluidity enhancement by electrical acupuncture stimulation is related to an adrenergic mechanism, J Acupunct Meridian Stud., 5(1): 21-28, 2012

[17] Kikuchi Y, Sato K, Ohki H. Optically accessible microchannels fomed in a single crystal silicon substrate for studics of blood rhcology.Microvasc Res., 44: 226-40, 1992

[18] Seki K, Sumino H, Murakami M. Study on blood rheology measured by MC-FAN. Rinsho Byori, 51(8): 770-5, 2003 (in Japanese) 
[19] Matsuno H, Tokuda H, Ishisaki A, Zhou Y, Kitajima Y, Kozawa O. P2Y12 receptors play a significant role in the development of platelet microaggregation in patients with diabetes. J Clin Endocrinol Metab., 90(2): 920-7, 2005:

[20] Yamamoto T, Kamei M, Yokoi N, Yasuhara T, Tei M, Kinoshita S. Platelet aggregates in various stages of diabetic retinopathy: evaluation using the particle-counting lightscattering method. Graefes Arch Clin Exp Ophthalmol, 243(7): 665-70, 2005

[21] Gao YZ, Yin QZ, Hisamitsu T, Jianf XH. An individual variation study of eectroacupuncture anagesia in rats using microarray. Am J Chinese Med., 35(5): 767-78, 2007

[22] Fukazawa Y, Maeda T, Hamabe W, Kumamoto K, Gao Y, Yamamoto C, Ozaki M, Kishioka S. Activation of Spinal Anti-analgesic System Following Electroacupuncture Stimulation in Rats. Journal of Pharmacological Sciences, 99(4): 408-14, 2005

[23] Uchida S. Acupuncture: Is there a physiological basis. Journal of Japan Society of Acupuncture and Moxibustion, 53(4): 555-60, 2003

[24] Okada K. Acupuncture stimulation and Analgesia. Proceedings of the Symposium on Biological and Physiological Engineering, 20: 223-4, 2005

[25] Murase K., Kawakita K. Diffuse noxious inhibitory controls in anti-nociception produced by acupuncture and moxibustion on trigeminal caudalis neurons in rats, Japanese J. Physiol., 50(1): 133-40, 2000

[26] Sumiya E, Kitade K. The theory of transcutaneus electrical nerva stimulation therapy and TENS stimulator. Treatment instruments for pain clinic, 30(2): 148-55, 2009

[27] Toda K. Mechanisms of descending antinociceptive system. Treatment instruments for pain clinic, 28(7): 975-87, 2007

[28] Wan J, Ristenpart WD, Stone HA. Dynamics of shear-induced ATP release from red blood cells. Proc Natl Acad Sci U S A., 105(43): 16432-7, 2008

[29] Hans Oberleithner. Nanophysiology of vascular endothelium. Kitasato Med. J., 40:97-102, 2010

[30] Higashi Y, Noma K, Yoshizumi M, Kihara Y. Endothelial function and oxidative stress in cardiovascular diseases. Circ J., 73(3):411-8, 2009

[31] Sugimoto M, Miyata S. Functional property of von Willebrand factor under flowing blood. Int J Hematol., 75(1):19-24, 2002

[32] Matsuoka I. ATP Receptor-mediated Cellular Responses and Their Regulation by Ecto, Nucleotidases, Fukushima Med. J. 53(2): 125-42, 2003

[33] Tang Y, Wang M, Chen C, Le X, Sun S, Yin Y. Cardiovascular protection with danshensu in spontaneously hypertensive rats. Biol Pharm Bull., 34(10):1596-601, 2011 